\title{
Sampling of Periodic Signals: A Quantitative Error Analysis
}

\author{
Mathews Jacob, Student Member, IEEE, Thierry Blu, Member, IEEE, and Michael Unser, Fellow, IEEE
}

\begin{abstract}
We present an exact expression for the $L_{2}$ error that occurs when one approximates a periodic signal in a basis of shifted and scaled versions of a generating function. This formulation is applicable to a wide variety of linear approximation schemes including wavelets, splines, and bandlimited signal expansions. The formula takes the simple form of a Parseval's-like relation, where the Fourier coefficients of the signal are weighted against a frequency kernel that characterizes the approximation operator. We use this expression to analyze the behavior of the error as the sampling step approaches zero. We also experimentally verify the expression of the error in the context of the interpolation of closed curves.
\end{abstract}

Index Terms-Asymptotic performance, curves, error bounds, periodic representations, sampling.

\section{INTRODUCTION}

C LASSICAL sampling theory deals with the problem of reconstructing or approximating a signal $s(t)$ from a set of uniform samples or measurements. In its generalized version, the reconstructed approximation [1] is

$$
s_{h}(t)=\sum_{k=-\infty}^{\infty} c_{k} \varphi\left(\frac{t}{h}-k\right)
$$

where the underlying basis functions are rescaled translates of the generating ${ }^{1}$ function $\varphi ; h$ is the sampling step. The generator can be selected to yield bandlimited (e.g., $\varphi=$ sinc), spline, or wavelet representations of signals. The expansion coefficients $c_{k}$ are either determined from the uniform samples of the input signal $s(k h)$ (interpolation or quasi-interpolation) or from a sequence of inner products with a suitable sequence of analysis functions [1]. This theory is well developed for the case in which the input signal is in $L_{2}(\mathbb{R})$, which also implies that it is defined over the whole real line. The approximation quality depends on the sampling step $h$, the type of algorithm used (e.g., interpolation versus projection), and, most importantly, on the choice of the generating function $\varphi$. This can be quantified rather precisely, thanks to the availability of sharp mean square error estimates in the $L_{2}(\mathbb{R})$ setting [3], [4]. Bounds are also available for the $L_{\infty}$ approximation error (worst-case scenario) [5].

Manuscript received April 5, 2001; revised January 8, 2002. This work was supported by the Swiss National Science Foundation under Grant 2100-053 540 The associate editor coordinating the review of this paper and approving it for publication was Dr. Helmut Boelcskei.

The authors are with the Biomedical Imaging Group, Swiss Federal Institute of Technology, Lausanne, Switzerland (e-mail: mathews.jacob@epfl.ch; thierry.blu@epfl.ch; michael.unser@epfl.ch).

Publisher Item Identifier S 1053-587X(02)03149-5.

${ }^{1}$ When the function satisfies a two-scale relation [2], it is called a scaling function (e.g., splines, Daubechies functions or sinc)
In this paper, we are interested in the case where the input signal $s(t)$ is periodic, which is an assumption that is commonly made in practice. One example, where the periodic representation is especially relevant, is the parametric representation of closed curves in terms of splines [7], [8], [9] or Fourier basis functions [10]. Assuming the period $T$ to be an integer multiple of the sampling step $(T=N h),{ }^{2}$ it is straightforward to adapt most of the $L_{2}$ techniques to the periodic case by simply considering periodized basis functions and by redefining the inner product accordingly [11] (see Section II). However, the error analysis for signals in $L_{2}(\mathbb{R})$ is not directly applicable because the square modulus of the Fourier transform is not defined for periodic signals.

The quantitative error analysis of periodic signals is the main focus of this paper. In particular, we will derive a general predictive error formula that depends on the Fourier coefficients of $s(t)$. Interestingly, the formula bears a strong resemblance to the error expression of signals in $L_{2}(\mathbb{R})$. However, the recipe is different although the ingredients are more or less the same as in [3]; the average least squares error is obtained as a discrete sum of the Fourier series coefficients, as opposed to a continuous integral in [3]. We also study the behavior of the approximation as the sampling step goes to zero.

\section{PREliminaries}

\section{A. Notations}

We denote the Fourier transform of a continuous signal $s(t)$ as

$$
\hat{s}(\omega)=\int_{-\infty}^{\infty} s(t) e^{-j \omega t} d t .
$$

\section{B. Sampling of Periodic Signals}

The general formula for determining the expansion coefficients in (1) is

$$
c_{k}=\int_{-\infty}^{\infty} s(\xi) \tilde{\varphi}\left(\frac{\xi}{h}-k\right) d \frac{\xi}{h}
$$

where $\tilde{\varphi}$ is an appropriate analysis function. The usual setting for this formula is $s \in L_{2}(\mathbb{R})$ (finite energy signals). In particular, one can show that $c_{k} \in \ell_{2}$ when $\hat{\tilde{\varphi}}$ is bounded and when $s$ has at least $r>1 / 2$ derivatives in the $L_{2}$ sense [3]. However, (3) also works for more general cases. For instance, if $s(t)$ is bounded, then the $c_{k}$ s will be bounded as well, provided that $\tilde{\varphi}$ is a distribution ${ }^{3}$ of order 0 .

\footnotetext{
${ }^{2}$ If we choose $T=N h$, the resulting representation is assured to be $T$ periodic. Otherwise, this property is not satisfied in general.

$3 \tilde{\varphi}$ is a distribution of order $n$ iff $|\langle\tilde{\varphi}, s\rangle| \leq C \max _{k<n} \sup _{x}\left|s^{(k)}(x)\right|$, where $C$ is a constant [12, pp. 24-25], [13, def. 1.3.1], e.g., the Dirac delta distribution $\delta(x)$ is of order 0 . An absolutely integrable function $\tilde{\varphi}$ can also be identified as a distribution of order 0 .
} 
We assume that $s(t)$ is $T$-periodic and that $T=N h$, where $N$ is a positive integer. Under those conditions, the sequence $c_{k}$ defined by (3) is periodic as well, with period $N$. Furthermore, we can rewrite the synthesis and analysis (1) and (3) using $N$-periodized functions as

$$
\begin{aligned}
s_{N}(t) & =\sum_{k=0}^{N-1} c_{k} \varphi_{p}\left(\frac{t}{h}-k\right) \\
c_{k} & =\int_{0}^{T} s(\xi) \tilde{\varphi}_{p}\left(\frac{\xi}{h}-k\right) d \frac{\xi}{h}
\end{aligned}
$$

where

$$
\varphi_{p}(t)=\sum_{l=-\infty}^{\infty} \varphi(t-l N) .
$$

Equation (5) calls for the definition of an inner product in $L_{2}([0, T])$. We denote the $L_{2}([0, T])$ inner product between two functions $s_{1}(t), s_{2}(t) \in L_{2}([0, T])$ as

$$
\left\langle s_{1}(t), s_{2}(t)\right\rangle_{L_{2}([0, T])}=\frac{1}{T} \int_{0}^{T} s_{1}(t) s_{2}(t) d t .
$$

The corresponding norm is written as $\|\cdot\|_{L_{2}([0, T])}$. We show in the Appendix A that a sufficient condition for $\varphi_{p}$ to be in $L_{2}([0, T])$ is that $\varphi$ be absolutely integrable $\left(\varphi \in L_{1}(\mathbb{R})\right)$ and that the discrete Fourier transform of the autocorrelation sequence

$$
\hat{a}_{\varphi}(\omega)=\sum_{k=-\infty}^{\infty}|\varphi(\omega+2 k \pi)|^{2}
$$

is bounded. Under those assumptions, $s_{N}(t) \in L_{2}([0, T])$ provided, of course, that the $c_{k} \mathrm{~s}$ are bounded. While these relatively mild conditions are satisfied by most generating functions used in practice, they are not applicable to the classical case $\varphi=\operatorname{sinc}$, which present some difficulties, i.e., sinc $\notin L_{1}(\mathbb{R})$. This case is dealt with in the next section.

Combining (4) and (5), we get

$$
\begin{aligned}
s_{N}(t) & =\mathcal{Q}_{N} s(t) \\
& =\sum_{k=0}^{N-1}\left[\int_{0}^{T} s(\xi) \tilde{\varphi}_{p}\left(\frac{\xi}{h}-k\right) d \frac{\xi}{h}\right] \varphi_{p}\left(\frac{t}{h}-k\right)
\end{aligned}
$$

where $\mathcal{Q}_{N}$ is the approximation operator. This linear operator is a projector if and only if the functions $\varphi$ and $\tilde{\varphi}$ are biorthogonal, i.e., $\langle\varphi(t-k), \tilde{\varphi}(t-l)\rangle=\delta_{k-l}$ [14]. In this case, $s_{N}(t)$ is a consistent reconstruction of the measurements $c_{k}$.

As we frequently use Parseval's relation, we now recall it. It relates the $L_{2}([0, T])$ inner product between two functions $s_{1}(t), s_{2}(t) \in L_{2}([0, T])$ to their Fourier series coefficients as

$$
\begin{aligned}
\left\langle s_{1}(t), s_{2}(t)\right\rangle_{L_{2}([0, T])} & =\frac{1}{T} \int_{0}^{T} s_{1}(t) s_{2}(t) d t \\
& =\sum_{k=-\infty}^{\infty} S_{1}(k) S_{2}(k)^{*} .
\end{aligned}
$$

Using this expression, the $L_{2}([0, T])$ norm of $s(t) \in L_{2}([0, T])$ can be written as

$$
\|s\|_{L_{2}([0, T])}^{2}=\frac{1}{T} \int_{0}^{T}|s(t)|^{2} d t=\sum_{k=-\infty}^{\infty}|S(k)|^{2} .
$$

\section{FOURIER SERIES REPRESENTATION}

Bandlimited periodic signals can be represented as (4) by choosing $\varphi=$ sinc. However, due to the slow decay of sinc, $\varphi_{p}$ does not converge when $N$ is even. However, when $N$ is odd, $\varphi_{p}$ converges to a well-defined function in $L_{1}([0, T])$. In this case, the signal representation can be reformulated as a Fourier series. Hence, we briefly review the Fourier series description of a periodic signal when the period is odd.

A $T$-periodic signal $\left(s(t) \in L_{2}([0, T])\right)$ can be expanded as

$$
s(t)=\sum_{k=-\infty}^{\infty} S(k) e^{j \frac{2 \pi k t}{T}}
$$

where the Fourier series coefficients $S(k)$ are obtained as

$$
S(k)=\frac{1}{T} \int_{0}^{T} s(t) e^{-j \frac{2 \pi k \cdot t}{T}} d t .
$$

In most practical applications, the function $s(t)$ is not directly available. Usually, it is only known through its samples $\{s(l h)\}_{l=0, \ldots N-1}$. In such cases, one often assumes that $s(t)$ is bandlimited and, hence, approximates the coefficients $S(k)$ with the $N$ point DFT of $\{s(l h)\}$ for $k=-\lfloor N / 2\rfloor \ldots\lfloor N / 2\rfloor$ and 0 otherwise.

The corresponding continuous signal $s_{N}(t)$ is nothing but the periodized sinc interpolation of the samples [15], [16]. The corresponding sinc interpolation with a zooming factor $M$ is implemented efficiently by computing the FFT of the input sequence and performing a larger size IFFT with zero padding the transform upto size $N M$. This representation turns out to be a special case of (9) with $\varphi=\operatorname{sinc}$ and $\tilde{\varphi}=\delta$-the Dirac's delta distribution.

\section{COMPuTATION OF THE SQuare ERror}

The space spanned by the generating functions is not shiftinvariant in general. Hence, the approximation error at a scale $h$ is dependent on a time shift of the function $s(t)$. The shifted function is denoted by $s_{\tau}(t)=s(t-\tau)$.

The mean square approximation error for a shifted function $s_{\tau}$ is given by

$$
\begin{aligned}
\gamma_{s}(\tau, N) & =\frac{1}{T} \int_{0}^{T}\left|s_{\tau}(t)-\mathcal{Q}_{N} s_{\tau}(t)\right|^{2} d t \\
& =\left\|s_{\tau}-\mathcal{Q}_{N} s_{\tau}(t)\right\|_{L_{2}([0, T])}^{2} .
\end{aligned}
$$

As the period of the signal is an integer multiple of the sampling step, $\gamma_{s}(\tau, N)$ is also $h$ periodic in $\tau$. In most applications, the exact phase of the signal is not known. Hence, we are interested in obtaining a measure of the error that is averaged over $\tau$. This average error is given by

$$
\eta_{s}(N)=\sqrt{\frac{1}{h} \int_{0}^{h} \gamma_{s}(\tau, N) d \tau} .
$$

The following theorem, which is the main result of this paper, gives an explicit expression for the mean error $\eta_{s}(N)$.

Theorem 1: Let $s(t)$ be a $T$-periodic signal with the Fourierseries coefficients $S(k)$. The mean square approximation error incurred in approximating $s(t)$ as in (9) is given by

$$
\eta_{s}(N)=\sqrt{\sum_{k=-\infty}^{\infty}|S(k)|^{2} E\left(\frac{2 \pi k}{N}\right)}
$$


where the approximation kernel $E(\omega)$ depends only on $\varphi$ and $\tilde{\varphi}$ and assumes the expression

$$
\begin{aligned}
E(\omega) & =\left|1-\hat{\tilde{\varphi}}(\omega)^{*} \hat{\varphi}(\omega)\right|^{2}+|\hat{\tilde{\varphi}}(\omega)|^{2} \sum_{n \neq 0}|\hat{\varphi}(\omega+2 n \pi)|^{2} \\
& =\underbrace{1-\frac{|\hat{\varphi}(\omega)|^{2}}{\hat{a}_{\varphi}(\omega)}}_{E_{\min }(\omega)}+\underbrace{\hat{a}_{\varphi}(\omega)\left|\hat{\tilde{\varphi}}(\omega)-\hat{\varphi}_{d}(\omega)\right|^{2}}_{E_{\mathrm{rcs}}(\omega)}
\end{aligned}
$$

where $\hat{\varphi}_{d}(\omega)=\left(\hat{\varphi}(\omega) / \hat{a}_{\varphi}(\omega)\right.$.

The proof is given in the Appendix B.

Note that this kernel is identical to the one obtained in the case of signals in $L_{2}(\mathbb{R})$ [3]. The main difference with the $L_{2}(\mathbb{R})$ case is that the expression of the error (16) is a discrete sum as opposed to a continuous integral [3]

$$
\eta_{s}(T)=\sqrt{\frac{1}{2 \pi} \int_{-\infty}^{\infty}|\hat{s}(\omega)|^{2} E(\omega T) d \omega} .
$$

Here, $\hat{s}(\omega)$ is the Fourier transform of the signal $s(t) \in L_{2}(\mathbb{R})$, and $T$ is the sampling step.

Given a reconstruction space, the error kernel attains its minimum possible value $E_{\min }(\omega)$ for all $\omega$ when $\tilde{\varphi}$ is the dual of $\varphi$. It is obvious from (18) as $E_{\text {res }}(\omega) \geq 0$, and $E_{\min }(\omega)$ depends only on $\varphi$. This case corresponds to the minimum error approximation (orthogonal projection), as in the case of signals in $L_{2}(\mathbb{R})$ [17]. The second part $E_{\text {res }}$ accounts for the additional error encountered for not choosing the optimal analysis function $\tilde{\varphi}=\varphi_{d}$. When $\tilde{\varphi}$ is bi-orthogonal to $\tilde{\varphi}$ but $\tilde{\varphi} \neq \varphi_{d}$, then the corresponding operator $\mathcal{Q}_{N}$ is called an oblique projection.

\section{Asymptotic PeRformance}

The asymptotic performance of the representation is determined by the behavior of the kernel close to the origin. Using the Taylor-series expression of the kernel, we show that for the minimum approximation error to decay as $\mathcal{O}\left(1 / N^{L}\right)$ as the number of sampling points $N \rightarrow \infty$, we need $\hat{\varphi}(0) \neq 0$ and $\hat{\varphi}^{(n)}(2 k \pi)=0, \forall k \in \mathbb{Z} \backslash\{0\}$ for $n=0,1 \ldots L-1$. These are precisely the Strang-Fix conditions of order $L$ [2]; a $\varphi$ that satisfies these conditions is called as an $L$ th-order generating function.

In the following theorem, we give the asymptotic bound for the projection error. Note that the projection need not be orthogonal [18].

Theorem 2: Let $\varphi$ and $\tilde{\varphi}$ be two mutually bi-orthogonal generating functions. Then, the oblique projection error in approximating an $L$-times differentiable function $s(t)$ as in (9) decays as $\mathcal{O}\left(1 / N^{L}\right)$ as $N \rightarrow \infty$ iff $\varphi$ is an $L$ th-order generating function. If $\varphi$ satisfies the $L$ th-order Strang-Fix conditions, the error in approximation as $N \rightarrow \infty$ is asymptotically given as

$$
\begin{aligned}
\eta_{s}(N) & =C_{\varphi, \tilde{\varphi}}\left\|(2 \pi k)^{L} S(k)\right\|_{\ell_{2}}\left(\frac{1}{N}\right)^{L}+\mathcal{O}\left(\frac{1}{N^{L+1}}\right) \\
& =C_{\varphi, \tilde{\varphi}} T^{L}\left\|s^{(L)}\right\|_{L_{2}[0, T)}\left(\frac{1}{N}\right)^{L}+\mathcal{O}\left(\frac{1}{N^{L+1}}\right)
\end{aligned}
$$

where $s^{(L)}$ is the $L$ th derivative of $s$, and the constant is given by the expression

$$
C_{\varphi, \tilde{\varphi}}=\frac{1}{L !} \sqrt{\sum_{k \neq 0}\left|\hat{\varphi}^{(L)}(2 \pi k)\right|^{2}+\left|m_{\varphi_{d}}^{L}-m_{\tilde{\varphi}}^{L}\right|^{2}} .
$$

Here, $\hat{\varphi}^{(L)}$ denotes the $L$ th derivative of $\varphi$, and $m_{u}^{L}=$ $\int x^{L} u(x) d x ; u$ is either $\tilde{\varphi}$ or $\varphi_{d}$.

The proof is given in Appendix C.

Note that this result is almost the same as the bound derived in [19], except that the present norm is defined for $L_{2}([0, T])$ as opposed to $L_{2}(\mathbb{R})$ as in [19]. The minimum value attainable by this constant $C_{\varphi}^{-}=(1 / L !) \sqrt{\sum_{k \neq 0}\left|\hat{\varphi}^{(L)}(2 \pi k)\right|^{2}}$ is independent of the analysis function. This value is achieved when we have $m_{\varphi_{d}}^{L}=m_{\tilde{\varphi}}^{L}$.

\section{EXPERIMENTAL VERIFICATION OF THE ERROR FORMULA}

In this section, we validate the expression for the error given by Theorem 1 experimentally. We compare the measured errors to the ones predicted by the theory for the approximation of a reference shape as a function of the sampling step $h$ or, equivalently, the number of the samples $N$.

Our reference shape (Switzerland) is polygonal with 807 edges and is represented using two periodic functions $x(t)$ and $y(t)$. For each experiment, the initial model $(x(t), y(t))$ was resampled to a specified number of points.

We considered two types of approximations: 1 ) a cubic spline interpolation with $\varphi=\beta^{3}$ (cubic spline) and 2) a bandlimited one with $\varphi=$ sinc. Note that the second approach is equivalent to a truncated Fourier approximation. In fact, we used an IFFT padded with zeros to generate the bandlimited interpolation functions at the required scale.

The comparisons between the experimental errors and the ones predicted by the theory are given in Figs. 1 and 2, respectively. It can be seen for both the graphs (Figs. 1 and 2) that the experimental error (for $\tau=0.5$ ) is in good agreement with the theoretical prediction. The experimentally obtained curve of $\gamma_{s}(\tau, N)$ for $\tau=0.5$ oscillates around the theoretically predicted curve of $\eta_{s}(N)$. This is because the theoretical prediction is an average of $\gamma_{s}(\tau, N)$ over all $\tau$ s.

From Fig. 3, it can be seen that the spline interpolation of curves perform slightly better (around $1 \mathrm{~dB}$ ) than the sinc interpolation. This behavior can be explained with the aid of the error kernel we have just derived. We can see from Fig. 4 that the spline kernel has lower values, as compared with the sinc interpolation kernel when $\omega>\pi$. Hence, at low sampling rates (when the signal has some nonnegligible frequency components above $\pi$ ), spline interpolation will usually outperform the sinc one. The differences tend to vanish as the sampling step decreases.

The map of Switzerland interpolated from 45 samples using the spline and sinc functions are shown in Fig. 5. It can be seen that at some places, the sinc representation results in looping curves. This effect is less likely with the spline representation due to the more local behavior of spline interpolation. 


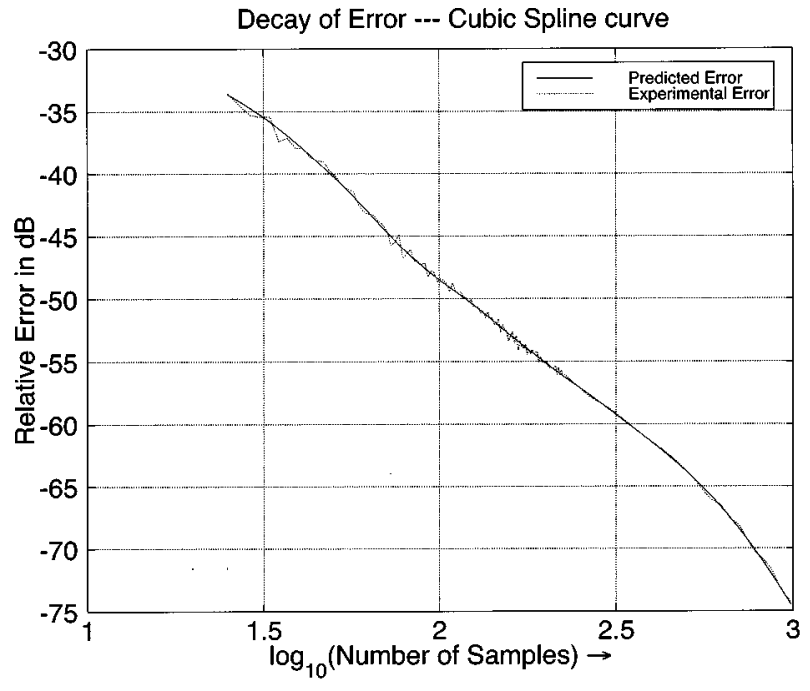

Fig. 1. Decay of the cubic spline interpolation error for the map of Switzerland as a function of the number of samples.

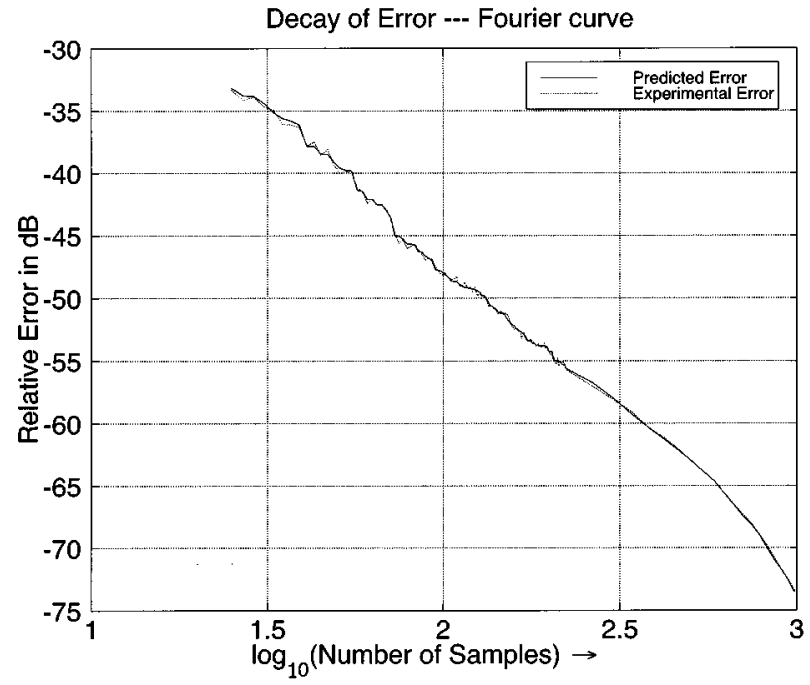

Fig. 2. Decay of the sinc interpolation error for the map of Switzerland as a function of the number of samples.
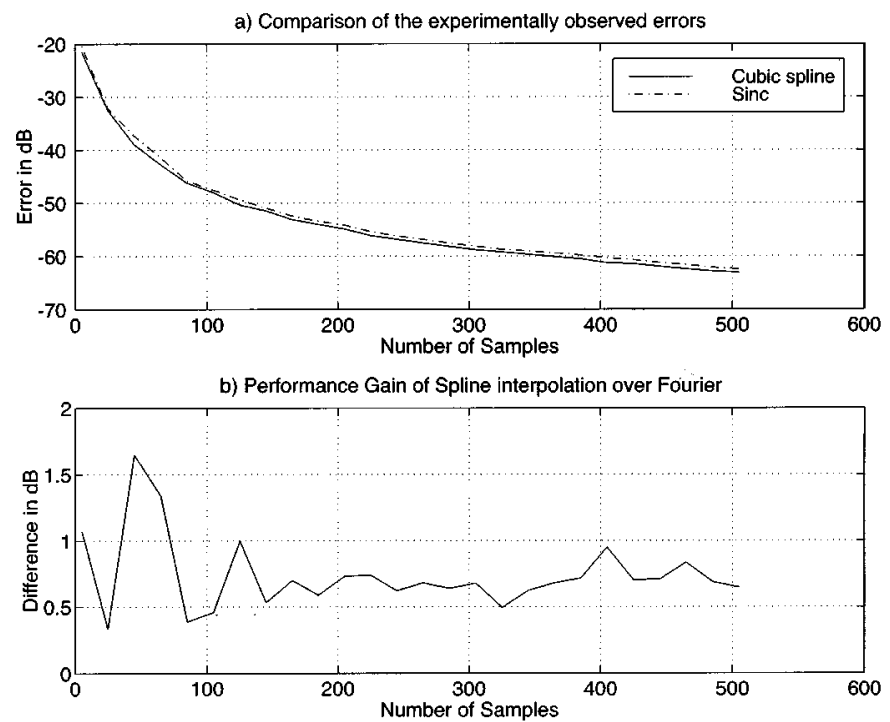

Fig. 3. Comparison of spline and sinc interpolation.

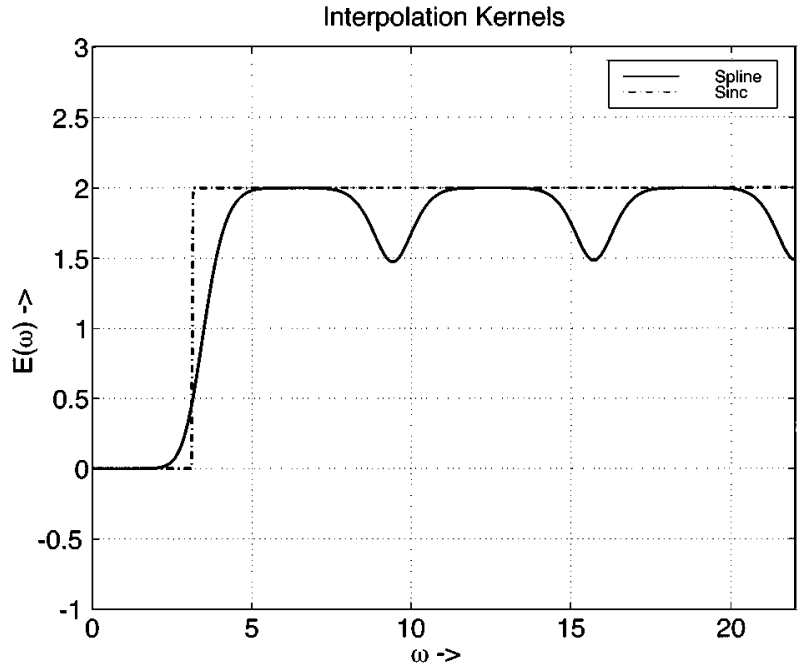

Fig. 4. Error kernels for cubic B-spline and sinc representation.

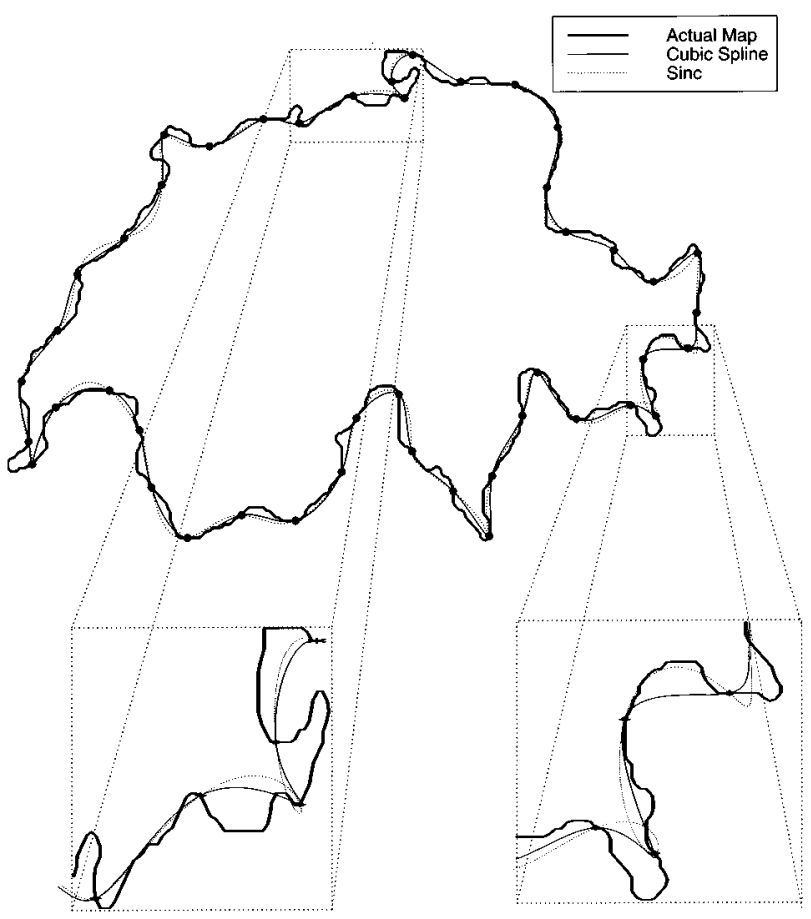

Fig. 5. Actual map of Switzerland represented using 807 edges is resampled to 45 points (indicated by dots). These points are then interpolated using cubic spline and sinc functions. The graphs below are the zoomed portions of the corresponding positions of the main graph, which illustrates the looping nature of sinc interpolation

\section{CONCLUSION}

We have derived an exact expression of the mean error in representing a periodic signal in a generating function basis. This expression may be useful for comparing different generating functions and for choosing the right one for an application. We have experimentally verified the expression; the experimental curves are in excellent agreement with the theoretical predictions. Using the expression for the error, we also analyzed the behavior of the approximation scheme as the sampling step approaches zero. 
APPENDIX A

SUFFICIENT CONDITION FOR $\varphi_{p} \in L_{2}([0, T])$

$\varphi \in L_{1}(\mathbb{R})$ implies that $\varphi_{p} \in L_{1}([0, T])$ and that

$\varphi_{p}(x)=\sum_{l \in \mathbb{Z}} \varphi(x-l N)=\frac{1}{N} \sum_{k \in \mathbb{Z}} \hat{\varphi}\left(\frac{2 k \pi}{N}\right) e^{j \frac{2 \pi k t}{N}}$

in the sense of distributions [20]. Now, the right-hand side of (22) is in $L_{2}([0, T])$ iff

$$
\sum_{k \in \mathbb{Z}}\left|\hat{\varphi}\left(\frac{2 k \pi}{N}\right)\right|^{2}<\infty
$$

which is ensured if the the Fourier transform of the autocorrelation

$$
\hat{a}_{\varphi}(\omega)=\sum_{k \in \mathbb{Z}}|\hat{\varphi}(\omega+2 k \pi)|^{2}
$$

is bounded for all $\omega$. Thus, $\varphi_{p} \in L_{2}([0, T])$.

\section{APPENDIX B}

COMPUTATION OF THE SQUARE ERROR

Expanding (14), we get

$$
\begin{aligned}
\gamma_{s}(\tau, N)= & \frac{1}{T} \int_{0}^{T}\left[s_{\tau}(t)\right]^{2} d t+\frac{1}{T} \int_{0}^{T}\left[\mathcal{Q}_{N} s_{\tau}(t)\right]^{2} d t \\
& -\frac{2}{T} \int_{0}^{T} s_{\tau}(t) \mathcal{Q}_{N} s_{\tau}(t) d t
\end{aligned}
$$

1) Using Parseval's theorem, the first term of (25) reduces to

$$
\frac{1}{T} \int_{0}^{T}\left[s_{\tau}(t)\right]^{2} d t=\sum_{k=-\infty}^{\infty}\left|S_{\tau}(k)\right|^{2}=\sum_{k=-\infty}^{\infty}|S(k)|^{2} .
$$

2) To compute the second term of (25), we first compute the Fourier coefficients of $\mathcal{Q}_{N} s_{\tau}(t)$. From (4), they are obtained as

$R_{N}(m)=\sum_{k=0}^{N-1} c_{k}\left[\frac{1}{T} \int_{0}^{T} \varphi_{p}\left(\frac{t}{h}-k\right) e^{-\frac{2 \pi m 2 t}{T}} d t\right]$

We make a change of variables as $t=t / h-k$ and rearrange the terms to get

$$
R_{N}(m)=\underbrace{\left[\int_{-\infty}^{\infty} \varphi(t) e^{-\frac{2 \pi m t}{N}} d t\right]}_{\varphi\left(\frac{2 \pi m}{N}\right)} \frac{1}{N} \sum_{k=0}^{N-1} c_{k} e^{\frac{2 \pi m k}{N}} .
$$

We now consider the expression of $c_{k}$ from (5); the $L_{2}([0, T])$ inner product can be expressed in terms of the corresponding Fourier coefficients using Parseval's theorem. Hence

$$
\begin{aligned}
\frac{1}{N} \sum_{k=0}^{N-1} c_{k} e^{\frac{2 \pi m k}{N}} & =\frac{1}{N} \sum_{k=0}^{N-1} \underbrace{\sum_{l=-\infty}^{\infty} S_{\tau}(l) \hat{\tilde{\varphi}}\left(\frac{2 \pi l}{N}\right)^{*} e^{-\frac{j 2 \pi k l}{N}} e^{\frac{2 \pi m k}{N}}}_{c_{k}} \\
& =\sum_{l=-\infty}^{\infty} S_{\tau}(l) \hat{\tilde{\varphi}}\left(\frac{2 \pi l}{N}\right)^{*} \underbrace{\sum_{k=0}^{\infty}}_{\sum_{k=-\infty}^{\infty} \sum_{k=-\infty} \delta_{(l-m-k N)}^{N-1} e^{\frac{j 2 \pi k(l-m)}{N}}} S_{\tau}(m+k N) \hat{\tilde{\varphi}}\left(\frac{2 \pi(m+k N)}{N}\right)^{*} .
\end{aligned}
$$

Combining (27) and (28), we get

$$
\begin{aligned}
R_{N}(m)= & \hat{\varphi}\left(\frac{2 \pi m}{N}\right) \\
& {\left[\sum_{k=-\infty}^{\infty} S_{\tau}(m+k N) \hat{\tilde{\varphi}}\left(\frac{2 \pi(m+k N)}{N}\right)^{*}\right] . }
\end{aligned}
$$

We now use Parseval's theorem to get

$$
\frac{1}{T} \int_{0}^{T}\left[\mathcal{Q}_{N} s_{\tau}(t)\right]^{2} d t=\sum_{m=-\infty}^{\infty}\left|R_{N}(m)\right|^{2} .
$$

Making use of the relation between the Fourier coefficients of the shifted function and the actual one $\left(S_{\tau}(k)=\right.$ $\left.e^{-j(2 \pi k \tau / T)} S(k)\right)$, we rewrite (29) as

$$
\begin{aligned}
\frac{1}{T} \int_{0}^{T}\left[\mathcal{Q}_{N} s_{\tau}(t)\right]^{2} & =\sum_{m=-\infty}^{\infty}\left|e^{-j \frac{2 \pi m \tau}{T}} x_{m}(\tau)\right|^{2}\left|\hat{\varphi}\left(\frac{2 \pi m}{N}\right)\right|^{2} \\
& =\sum_{m=-\infty}^{\infty}\left|x_{m}(\tau)\right|^{2}\left|\hat{\varphi}\left(\frac{2 \pi m}{N}\right)\right|^{2} .
\end{aligned}
$$

Here, $x_{m}(\tau)$ is the $h=(T / N)$ periodic function with the expression

$$
x_{m}(\tau)=\sum_{k=-\infty}^{\infty} \underbrace{S(m+k N) \hat{\varphi}\left(\frac{2 \pi(m+k N)}{N}\right)}_{X_{m}(k)} e^{-j \frac{2 \pi k \tau}{h}}
$$

Averaging this expression over $\tau,(1 / h) \int_{0}^{h}(d \tau / T) \int_{0}^{T}$ $\left|\mathcal{Q}_{N} s_{\tau}(t)\right|^{2} d t$ becomes

$$
\begin{aligned}
\sum_{m=-\infty}^{\infty}\left|\hat{\varphi}\left(\frac{2 \pi m}{N}\right)\right|^{2} \cdot[ & {\left[\frac{1}{h} \int_{0}^{h}\left|x_{m}(\tau)\right|^{2} d \tau\right] } \\
& =\sum_{m=-\infty}^{\infty}\left|\hat{\varphi}\left(\frac{2 \pi m}{N}\right)\right|^{2} \sum_{k=-\infty}^{\infty}\left|X_{m}(k)\right|^{2} .
\end{aligned}
$$

Here, we again made use of Parseval's theorem. Substituting for $X_{m}(k)$ and making a change of variable, the above summation can be rewritten as

$$
\begin{aligned}
& \frac{1}{h} \int_{0}^{h} \frac{1}{T} d \tau \int_{0}^{T} \mid \mathcal{Q}_{N} s_{\tau}(t)^{2} d t \\
&=\sum_{k}|S(k)|^{2} \hat{a}_{\varphi}\left(\frac{2 \pi k}{N}\right)\left|\hat{\tilde{\varphi}}\left(\frac{2 \pi k}{N}\right)\right|^{2}
\end{aligned}
$$


3) Making use of (29) and the Parseval's relation, we rewrite the third integral $(1 / T) \int_{0}^{T} s_{\tau}(t)^{*} \mathcal{Q}_{N} s_{\tau}(t) d t$ as

$\sum_{m} \underbrace{S_{\tau}(m)^{*} \hat{\varphi}\left(\frac{2 \pi m}{N}\right)^{*}}_{\left[S^{*}(m) \hat{\varphi}\left(\frac{2 \pi m}{N}\right)^{*}\right]^{j \frac{2 \pi m \tau}{T}}} \underbrace{\sum_{k} S_{\tau}(m+k N) \hat{\varphi}\left(\frac{2 \pi(m+k N)}{N}\right)^{*}}_{e^{-j \frac{2 \pi m \tau}{T}} x_{m}(\tau)}$.

Rearranging the terms, we get

$\frac{1}{T} \int_{0}^{T} s_{\tau}(t)^{*} \mathcal{Q}_{N} s_{\tau}(t) d t=\sum_{m}\left[S(m)^{*} \hat{\varphi}\left(\frac{2 \pi m}{N}\right)^{*}\right] x_{m}(\tau)$.

As before, $x_{m}(\tau)$ is a sequence of $h$ periodic functions. Now, averaging over $\tau$ as before, the term $(1 / h) \int_{0}^{h}(d \tau / T) \int_{0}^{T} s_{\tau}(t)^{*} \mathcal{Q}_{N} s_{\tau}(t) d t$ becomes

$$
\sum_{m}\left[S(m)^{*} \hat{\varphi}\left(\frac{2 \pi m}{N}\right)^{*}\right] \cdot \underbrace{\frac{1}{h} \int_{0}^{h} x_{m}(\tau) d \tau}_{X_{m}(0)=S(m) \hat{\varphi}\left(\frac{2 \pi m}{N}\right)^{*}} .
$$

Substituting for the expression of $X_{m}(0)$ the expression above reduces to

$$
\sum_{m}|S(m)|^{2} \hat{\tilde{\varphi}}\left(\frac{2 \pi m}{N}\right)^{*} \hat{\varphi}\left(\frac{2 \pi m}{N}\right)
$$

which is equivalent to

$$
\sum_{m}|S(m)|^{2} \Re\left(\hat{\tilde{\varphi}}\left(\frac{2 \pi m}{N}\right)^{*} \hat{\varphi}\left(\frac{2 \pi m}{N}\right)\right) .
$$

Combining the three integrals, we get

$$
\eta_{s}(N)=\sqrt{\sum_{k=-\infty}^{\infty}|S(k)|^{2} E\left(\frac{2 \pi k}{N}\right)}
$$

where

$$
\begin{aligned}
E(\omega) & =1+a_{\varphi}(\omega)|\hat{\tilde{\varphi}}(\omega)|^{2}-2 \Re(\hat{\tilde{\varphi}}(\omega) \hat{\varphi}(\omega)) \\
& =|1-\hat{\tilde{\varphi}}(\omega) \hat{\varphi}(\omega)|^{2}+|\hat{\tilde{\varphi}}(\omega)|^{2} \sum_{k \neq 0}|\hat{\varphi}(\omega+2 n \pi)|^{2}
\end{aligned}
$$

\section{APPENDIX C}

\section{ASYMPTOTIC PERFORMANCE}

In this proof, we assume that the kernel is $L$ times continuously differentiable. Initially, we derive the conditions for which $\lim _{N \rightarrow \infty}\left(\eta_{s}(N)\right)^{2}=0$. As $E(\omega)$ is bounded and $s(t) \in L_{2}([0, T])$, we use Lebesgue's dominated convergence theorem to interchange the limit and the summation in (16) to obtain

$$
\begin{aligned}
\lim _{N \rightarrow \infty}\left(\eta_{s}(N)\right)^{2} & =\sum_{k \in \mathbb{Z}}|S(k)|^{2} \lim _{N \rightarrow \infty} E\left(\frac{2 \pi k}{N}\right) \\
& =\sum_{k \in \mathbb{Z}}|S(k)|^{2} E(0)=0 .
\end{aligned}
$$

Here, we used the continuity of the kernel. The above expression is true for any $s(t) \in L_{2}([0, T])$ if $E(0)=0$. We have

$$
E(0)=\frac{1}{a_{\varphi}(0)} \sum_{l \neq 0}|\hat{\varphi}(2 l \pi)|^{2}+a_{\varphi}(0)\left|\hat{\tilde{\varphi}}(0)-\hat{\varphi}_{d}(0)\right|^{2}=0 .
$$

As the expression is a sum of positive quantities, it is equal to zero only if each of them is zero independently. In particular, we need $\hat{\varphi}(2 l \pi)=0, l \in \mathbb{Z} \backslash\{0\}$ and $\hat{\tilde{\varphi}}(0)=\hat{\varphi}_{d}(0)$. We also need $a_{\varphi}(0) \neq 0$, which is true iff $\hat{\varphi}(0) \neq 0$. These are precisely the Strang-Fix conditions of order 1.

Now, we look at the conditions for $\lim _{N \rightarrow \infty}\left(N \eta_{s}(N)\right)^{2}=$ 0 . This will imply that $\eta_{s}(N)$ decays faster than $\mathcal{O}(1 / N)$ as $N \rightarrow \infty$. To derive the conditions, we rewrite the expression for $N \eta_{s}(N)$ as

$$
\left(N \eta_{s}(N)\right)^{2}=\sum_{k \in \mathbb{Z}}|S(k)(2 k \pi)|^{2} \frac{N^{2}}{(2 k \pi)^{2}} E\left(\frac{2 \pi k}{N}\right) .
$$

Now, computing the limits by interchanging the sum and limit as $\left(E(\omega) / \omega^{2}\right)$ is bounded, we get

$$
\lim _{N \rightarrow \infty}\left(N \eta_{s}(N)\right)^{2}=\underbrace{\|S(k)(2 k \pi)\|_{\ell_{2}}^{2}}_{=T^{2}\left\|s^{(1)}\right\|_{L_{2}[0, T)}^{2}} \lim _{\omega \rightarrow 0}\left(\frac{E(\omega)}{\omega^{2}}\right) .
$$

Here, we made use of the fact that $E(\omega)$ is an even function of $\omega$ (its Taylor series has only even powers of $\omega$ ).

$$
\begin{aligned}
\lim _{\omega \rightarrow 0}\left(\frac{E(\omega)}{\omega^{2}}\right)= & \frac{1}{a_{\varphi}(0)} \sum_{l \neq 0}\left|\lim _{\omega \rightarrow 0} \frac{\varphi(\omega+2 l \pi)}{\omega}\right|^{2} \\
& +a_{\varphi}(0)\left|\lim _{\omega \rightarrow 0} \frac{\hat{\tilde{\varphi}}(\omega)}{\omega}-\lim _{\omega \rightarrow 0} \frac{\hat{\varphi}_{d}(\omega)}{\omega}\right|^{2} \\
= & \sum_{l \neq 0}\left|\frac{\varphi^{1}(2 l \pi)}{1 !}\right|^{2}+\left|\frac{\hat{\tilde{\varphi}}^{(1)}(0)-\hat{\varphi}_{d}^{(1)}(0)}{1 !}\right|^{2} .
\end{aligned}
$$

With the same argument as before, in addition to Strang-Fix conditions of order 1 , we need $\varphi^{(1)}(2 l \pi)=0, l \in \mathbb{Z} \backslash\{0\}$ and $\hat{\tilde{\varphi}}^{(1)}(0)=\hat{\varphi}_{d}^{(1)}(0)$. Continuing in the same fashion, we can see that $\eta_{S}(N)$ will decay as $\mathcal{O}\left(1 / N^{L}\right)$ iff $\varphi$ is an $L$ th-order generating function, and $\hat{\tilde{\varphi}}^{m)}(0)=\hat{\varphi}_{d}^{(m)}(0)$ for $m=0 \ldots L-$ 1.

The function $\hat{\varphi}_{d}(\omega)=\left(\hat{\varphi}(\omega) / \sum_{k}|\hat{\varphi}(\omega+2 k \pi)|^{2}\right)$ behaves as $\hat{\varphi}_{d}(\omega)=\left(1 / \hat{\varphi}^{*}(\omega)\right)+\mathcal{O}(\omega)^{L}$ as $\omega \rightarrow 0$. Since $\hat{\varphi}$ is bi-orthogonal to $\hat{\varphi}$, it behaves as $\hat{\tilde{\varphi}}(\omega)=\left(1 / \hat{\varphi}^{*}(\omega)\right)+\mathcal{O}(\omega)^{L}$ as $\omega \rightarrow 0$. (This follows from the bi-orthogonality relation $\sum_{k \in \mathbb{Z}} \hat{\varphi}(\omega+2 k \pi) \hat{\tilde{\varphi}}(\omega+2 k \pi)=1$.) Hence, $\tilde{\varphi}$ being bi-orthogonal to $\varphi$ ensures that $\hat{\tilde{\varphi}}^{m)}(0)=\hat{\varphi}_{d}^{(m)}(0)$ for $m=0 \ldots L-$ 1. Thus, the bi-orthogonality and the Strang-Fix conditions of order $L$ are sufficient for the error $\eta_{s}(N)$ to decay as $\mathcal{O}\left(1 / N^{L}\right)$.

$L$ is the first positive integer for which

$$
\begin{aligned}
\lim _{N \rightarrow \infty}\left(N^{L} \eta_{s}(N)\right)^{2} & =\underbrace{\|S(k)(2 k \pi)\|_{\ell_{2}}^{2}}_{=T^{2 L}\left\|_{s}(L)\right\|_{L_{2}[0, T)}^{2}} \underbrace{\lim _{\omega \rightarrow 0}\left(\frac{E(\omega)}{\omega^{2 L}}\right)}_{=\left(C_{\varphi}, \tilde{\varphi}\right)^{2}} \\
& \neq 0 .
\end{aligned}
$$

Proceeding as in (32), the expression of $C_{\varphi, \tilde{\varphi}}$ is

$$
C_{\varphi, \tilde{\varphi}}=\sqrt{\sum_{k \neq 0}\left|\frac{\hat{\varphi}^{(L)}(2 k \pi)}{L !}\right|^{2}+\left|\frac{m_{\hat{\varphi}}^{L}-m_{\hat{\varphi}_{d}}^{L}}{L !}\right|^{2}} .
$$


In the above equation, we substituted for $\hat{\tilde{\varphi}}^{(L)}(0)$ and $\hat{\varphi}_{d}^{(L)}(0)$ with $(-j)^{L} m_{\tilde{\varphi}}^{L}$ and $(-j)^{L} m_{\varphi_{d}}^{L}$, respectively, where $m_{u}^{L}=\int x^{L} u(x) d x$.

\section{REFERENCES}

[1] M. Unser, "Sampling-50 years after shannon," Proc. IEEE, vol. 88, pp. $569-587,2000$.

[2] G. Strang and T. Q. Nguyen, Wavelets and Filter Banks. Wellesley, MA: Wellesley-Cambridge, 1996.

[3] T. Blu and M. Unser, "Quantitative Fourier analysis of approximation techniques: Part I-Interpolators and projectors,” IEEE Trans. Signal Processing, vol. 47, pp. 2783-2795, Oct. 1999.

[4] _ , "Quantitative Fourier analysis of approximation techniques: Part II-Wavelets," IEEE Trans. Signal Processing, vol. 47, pp. 2796-2806, Oct. 1999.

[5] A. J. E. M. Janssen, "The Zak transform and sampling theorems for wavelet subspaces," IEEE Trans. Signal Processing, vol. 41, pp. 3360-3364, Dec. 1992.

[6] P. Brigger, J. Hoeg, and M. Unser, "B-spline snakes: A flexible tool for parametric contour detection," IEEE Trans. Image Processing, vol. 9, pp. 1484-1496, Sept. 2000.

[7] F. S. Cohen and J. Y. Wang, "Modeling image curves using invariant 3-D object curve models, a path to 3-D recognition and shape estimation from image contours, part 1," IEEE Trans. Pattern Anal. Machine Intell., vol. 16, pp. 1-12, Jan. 1994.

[8] R. H. Bartels, J. C. Beatty, and B. A. Barsky, An Introduction to Splines for Use in Computer Graphics and Geometric Modeling. San Mateo, CA: Morgan Kauffmann, 1987.

[9] Z. Huang and F. S. Cohen, "Affine-invariant B-spline moments for curve matching," IEEE Trans. Image Processing, vol. 5, pp. 1473-1480, Oct. 1996.

[10] G. H. Granlund, "Fourier preprocessing for hand print character recognition," IEEE Trans. Comput., vol. C-21, pp. 195-201, 1972.

[11] G. C. H. Chuang and J. Kuo, "Wavelet descriptor of planar curves: Theory and applications," IEEE Trans. Image Processing, vol. 5, pp. 56-70, Jan. 1996.

[12] L. Schwartz, Theorie des Distributions. Paris, France: Hermann, 1988

[13] G. Friedlander and M. Joshi, Introduction to the Theory of Distributions. Cambridge, U.K.: Cambridge Univ. Press, 1988.

[14] M. Unser and A. Aldroubi, "A general sampling theorem for nonideal acquistion devices," IEEE Trans. Signal Process., vol. 42, pp. 2915-2925, Nov. 1994

[15] F. Candocia and J. C. Prince, "Comments in sinc interpolation of discrete periodic signals," IEEE Trans. Signal Processing, vol. 46, pp. 2044-2047, July 1998.

[16] S. R. Doodey and A. K. Nandi, "Notes on interpolation of discrete periodic signals using sinc function related approaches," IEEE Trans. Signal Processing, vol. 48, pp. 1201-1203, Apr. 2000.

[17] A. Aldroubi and M. Unser, "Sampling procedures in function spaces and asymptotic equivalence with Shannon's sampling theory," Numer. Funct. Anal. Opt., vol. 42, pp. 1-21, 1994.

[18] M. Unser and A. Aldroubi, "A general sampling theory for nonideal acquisition devices," IEEE Trans. Signal Processing, vol. 42, pp. 2915-2925, Nov. 1994.

[19] M. Unser, "Approximation power of biorthogonal wavelet expansions," IEEE Trans. Signal Processing, vol. 44, pp. 519-527, Mar. 1996.

[20] T. Blu and M. Unser, "Approximation error for quasiinterpolators and multi-wavelet expansions," Appl. Comput. Harmon. Anal., vol. 6, pp. 219-251, 1999

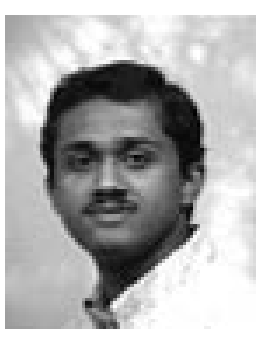

Mathews Jacob (S'00) was born in Kerala, India, in 1975. He received the M.E. degree in signal processing from the Indian Institute of Science, Bangalore, in 1999.

Currently, he is a Research Assistant with the Biomedical Imaging Group at the Swiss Federal Institute of Technology (EPFL), Lausanne, Switzerland. His research interests include image processing, active contour models, sampling theory, etc.

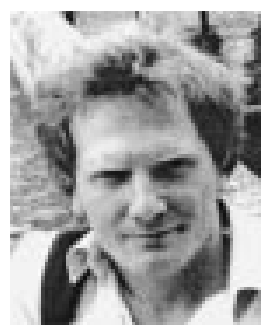

Thierry Blu (M'96) was born in Orléans, France, in 1964. He received the Diplôme d'ingénieur degree from École Polytechnique, Paris, France, in 1986 and from Télécom Paris (ENST) in 1988. He received the $\mathrm{Ph} . \mathrm{D}$ degree in electrical engineering in 1996 from ENST for a study on iterated rational filterbanks applied to wideband audio coding.

$\mathrm{He}$ is currently with the Biomedical Imaging Group at the Swiss Federal Institute of Technology (EPFL), Lausanne, on leave from France Télécom National Centre for Telecommunication Studies (CNET), Issy-les-Moulineaux, France. His interests include (multi)wavelets, multiresolution analysis, multirate filterbanks, approximation and sampling theory, and psychoacoustics.

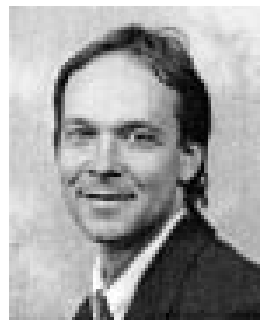

Michael Unser (F'99) was born in Zug, Switzerland, on April 9, 1958. He received the M.S. (summa cum laude) and Ph.D. degrees in electrical engineering in 1981 and 1984, respectively, from the Swiss Federal Institute of Technology (EPFL), Lausanne.

From 1985 to 1997, he was with the Biomedical Engineering and Instrumentation Program, National Institutes of Health, Bethesda, MD, where he headed the Image Processing Group. He is now Professor and Head of the Biomedical Imaging Group at EPFL. His main research area is biomedical image processing. He has a strong interest in sampling theories, multiresolution algorithms, wavelets, and the use of splines for image processing. He is the author of over 90 published journal papers in these areas. He is on the editorial boards of Signal Processing, the Journal of Visual Communication and Image Representation, Sampling Theory in Signal and Image Processing, and Pattern Recognition.

Dr. Unser is an Associate Editor for the IEEE TRANSACTIONS ON MEDICAL IMAGING and is a Guest Editor for its special issue on Wavelets in Medical Imaging. He was a member of the Image and Multidimensional Signal Processing Committee of the IEEE Signal Processing Society from 1993 to 2000 and was former Associate Editor for the IEEE TRANSACTIONS ON IMAGE PROCESSING from 1992 to 1995 and of the IEEE SIGNAL PROCESSING LETTERS from 1994 to 1998. He co-organized the 1994 IEEE-EMBS Workshop on Wavelets in Medicine and Biology and serves as regular conference chair for SPIE's Wavelet Applications in Signal and Image Processing, which has been held annually since 1993 . He is general co-chair for the IEEE International Symposium on Biomedical Imaging (ISBI'2002), which is a new conference to be held in Washington, DC, from July 7 to 10, 2002. He received the Dommer prize for excellence from EPFL in 1981, the research prize of the Brown-Boveri Corporation (Switzerland) for his thesis in 1984, the IEEE Signal Processing Society's 1995 Best Paper Award, and IEEE Signal Processing Society's 2000 Magazine Award. He is a member of IEEE, EURASIP, SPIE, and SIAM. 